\title{
Minimizing Radiation Exposure from Patients Treated with lodine-131 for Hyperthyroidism Using a Lead Collar: A Simple and Effective Approach
}

\author{
Issa Loutfi Mohamed Sakr Awatef M. Al-Shummari \\ Department of Nuclear Medicine, Faculty of Medicine, Kuwait University, Kuwait
}

\section{Key Words}

lodine-131 · Treatment · Hyperthyroidism $\cdot$ Radiation protection $\cdot$ Shielding

\begin{abstract}
Objective: To construct a lead collar useful for shielding the electromagnetic radiation from the thyroid of patients treated with radioiodine ${ }^{131}$ I for hyperthyroidism, thereby reducing the radiation emitted from these patients. Materials and Methods: A specially designed cervical lead collar was used to shield radiation exposure from 'hot thyroids' of 20 patients treated with iodine-131 for hyperthyroidism. The collar was made of lead strips stacked together around a plastic neck support fastened around the patient's neck. Measurements of the radiation exposure rate were obtained at increasing distances from the patients $(50,100,200 \mathrm{~cm})$, with and without the lead collar, at various times after ${ }^{131}$ I treatment. Results: Radiation exposure measurements over 3 weeks showed relatively high exposure rates in the first week after treatment. The radiation exposure from the patients wearing the lead collar was half that found in the same patients not wearing the collar. Conclusion: The use of the lead collar significantly reduced the radiation exposure of patients' surroundings. It was particularly useful in patients who could not abide strictly to the standard restrictive protocols after treatment with ${ }^{131}$ I.
\end{abstract}

Copyright @ 2003 S. Karger AG, Basel

\section{Introduction}

The use of iodine-131 for the treatment of hyperthyroidism has been increasing in recent years [1]. This treatment modality is effective in controlling hyperthyroidism, simple to administer and widely available at relatively low cost. Two main drawbacks are associated with radioiodine treatment: (1) radiation from the patient to the immediate family members and the general public [25], (2) iatrogenic induction of hypothyroidism particularly in patients with Graves' disease [6].

Radiation from patients treated with ${ }^{131}$ I has been studied extensively, particularly in comparison to the radiation dose limits to members of the public set by various regulatory bodies. The generally accepted limits range from 1 to $5 \mathrm{mSv} /$ year $(0.1-0.5 \mathrm{rem} /$ year) [7-11]. Further reduction of the dose equivalent limit is recommended in pregnant women and young children [12-14]. Thus, in order to minimize potential harmful effects of ${ }^{131}$ I irradiation and to comply with the rules regulating the limits of radiation dose, a number of protocols have been developed. These protocols were based on calculations of the radiation exposure from the subjects [15] or actual dosimetric measurements in the relatives and the immediate surroundings of the patient $[16,17]$. The protective measures rely in most cases on the relative isolation of the patient receiving the ${ }^{131} \mathrm{I}$ treatment especially restricting the contact with children and pregnant women in addi-

Dr. Issa Loutfi

Department of Nuclear Medicine

Faculty of Medicine, Kuwait University, PO Box 24923

Safat 13110 (Kuwait)

Tel. +965 5319592, Fax +965 5338936, E-Mail loutfi@hsc.kuniv.edu.kw 

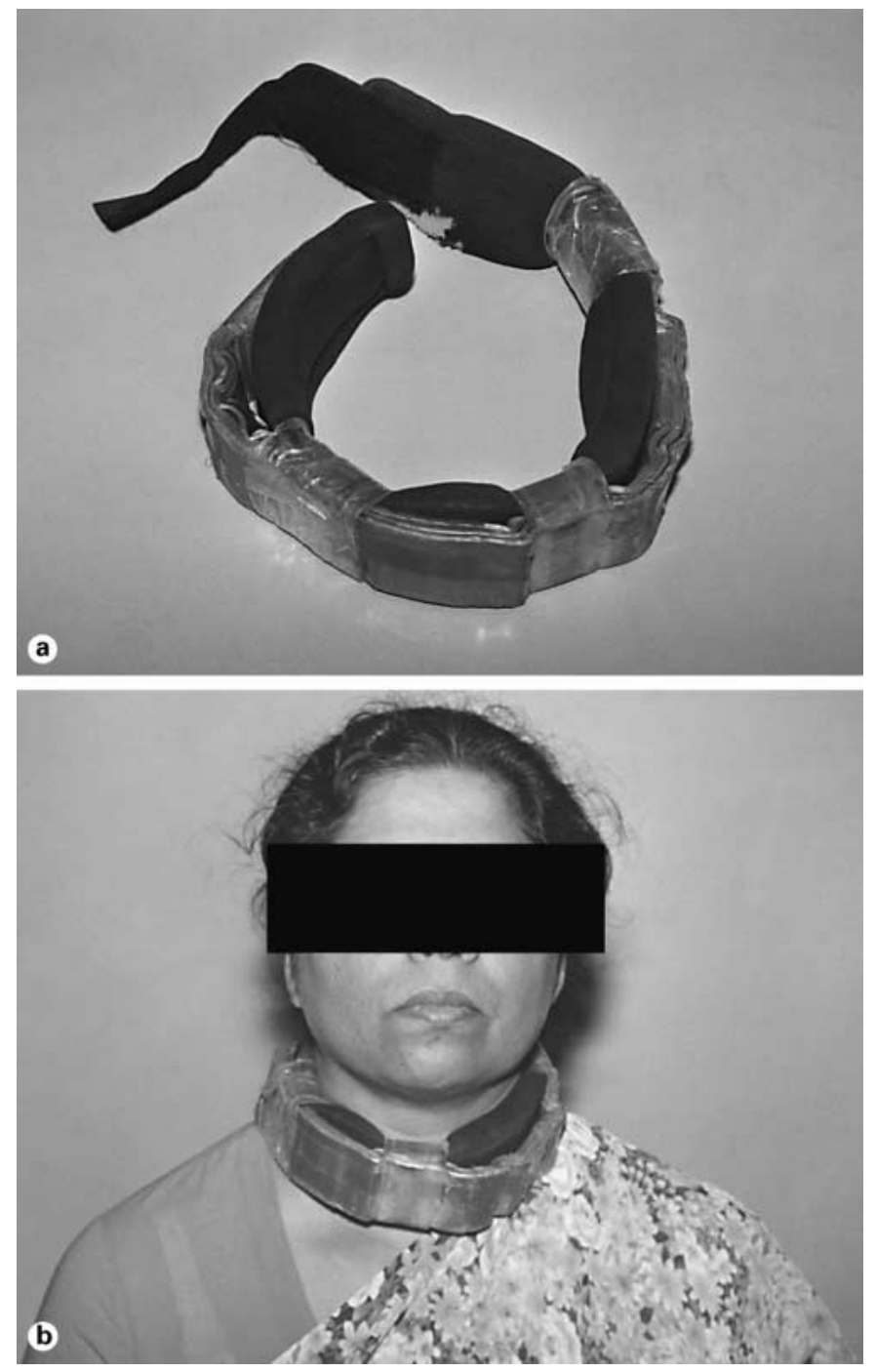

Fig. 1. a The lead collar assembly. b The lead collar as worn around the neck.

Table 1. Example of the radiation protection instructions to be followed by patients treated with ${ }^{131}$ I for hyperthyroidism in our institution

Department of Nuclear Medicine

Instructions to Patients Receiving ${ }^{131}$ I Therapy for Hyperthyroidism

Sleep alone in a separate room for 1 week especially if children are in the house

Use a separate toilet if available; flush toilet 3 times after use Wash out bathtub with soap or cleanser thoroughly after use Wash bed linen and clothing separately in a washing machine Females should use contraception to avoid pregnancy for 3 months after treatment; breast-feeding should be stopped tion to hygienic measures related to disposal of excreted radioactivity in urine, sweat and the gastrointestinal tract [18]. An example of such a protocol, which is used at our institution, is shown in table 1.

The study of the biological behavior of ${ }^{131}$ I radioactivity in treated hyperthyroid patients confirms that the main source of radiation from these patients is gamma rays emitted from the thyroid gland [19, 20]. In most cases, localization of the administered ${ }^{131} \mathrm{I}$ in the thyroid is completed by $24 \mathrm{~h}$. The variable amount of radioactivity (10$90 \%$ ) localized in the gland depends on the size and functional status of the gland. Subsequently, the elimination of the radioactivity is gradual, lasting usually over 2-3 weeks depending on the effective half-life of the radiotracer in the thyroid [21].

Based on the observation that the thyroid is the main source of irradiation from these patients, we decided to develop a simple method for minimizing the radiation exposure emitted by the patients.

\section{Subjects and Methods}

\section{Subjects}

The study involved 20 patients with hyperthyroidism referred for radioiodine treatment over a period of 2 years (average age 37 years, range $18-51$ years, F:M ratio 5.7). Radioiodine therapy was administered orally as a solution of ${ }^{131}$ I sodium iodide (Amersham Health, UK) in drinking water. The amount of ${ }^{131}$ I radioactivity given (296$851 \mathrm{MBq}, 8-23 \mathrm{mCi}$ ) was calculated for each patient based on the clinically estimated thyroid size and the 24-hour radioiodine uptake (range 14-95\%). The patients were asked to fast for $3 \mathrm{~h}$ before and after the administration of the radiopharmaceutical. In female patients of child-bearing age, a negative pregnancy test was documented, and the patient was instructed not to become pregnant for 3 months after the treatment. None of the women treated was breast feeding. An informed consent was obtained from each patient for participation in the study. In addition, all patients were instructed to follow the instructions of radiation protection as shown in table 1.

\section{The Lead Collar}

The lead collar consisted of synthetic sponge-like material (of the type used to support the neck after trauma) to which strips of lead foil (Biodex Medical Systems Inc., USA) were fixed to provide shielding from the radiation. The strips - each $50 \mathrm{~cm}$ long, $4 \mathrm{~cm}$ wide and $0.3 \mathrm{~mm}$ thick - were cut from a roll of lead foil. Fourteen strips were stacked and wrapped together to form a single strip of lead weighing $1.4 \mathrm{~kg}$. The resulting flexible lead strip was then affixed to the outside surface of the neck collar using adhesive tape (fig. 1). Each patient was asked to wear the collar-lead assembly snugly around the neck fastening it at the back using a velcro strap in the soft collar part of the device.

\section{Measurement of Radiation Exposure}

Measurements were performed at various times up to 3 weeks after ${ }^{131} \mathrm{I}$ treatment with the patient in a sitting position at 50,100 


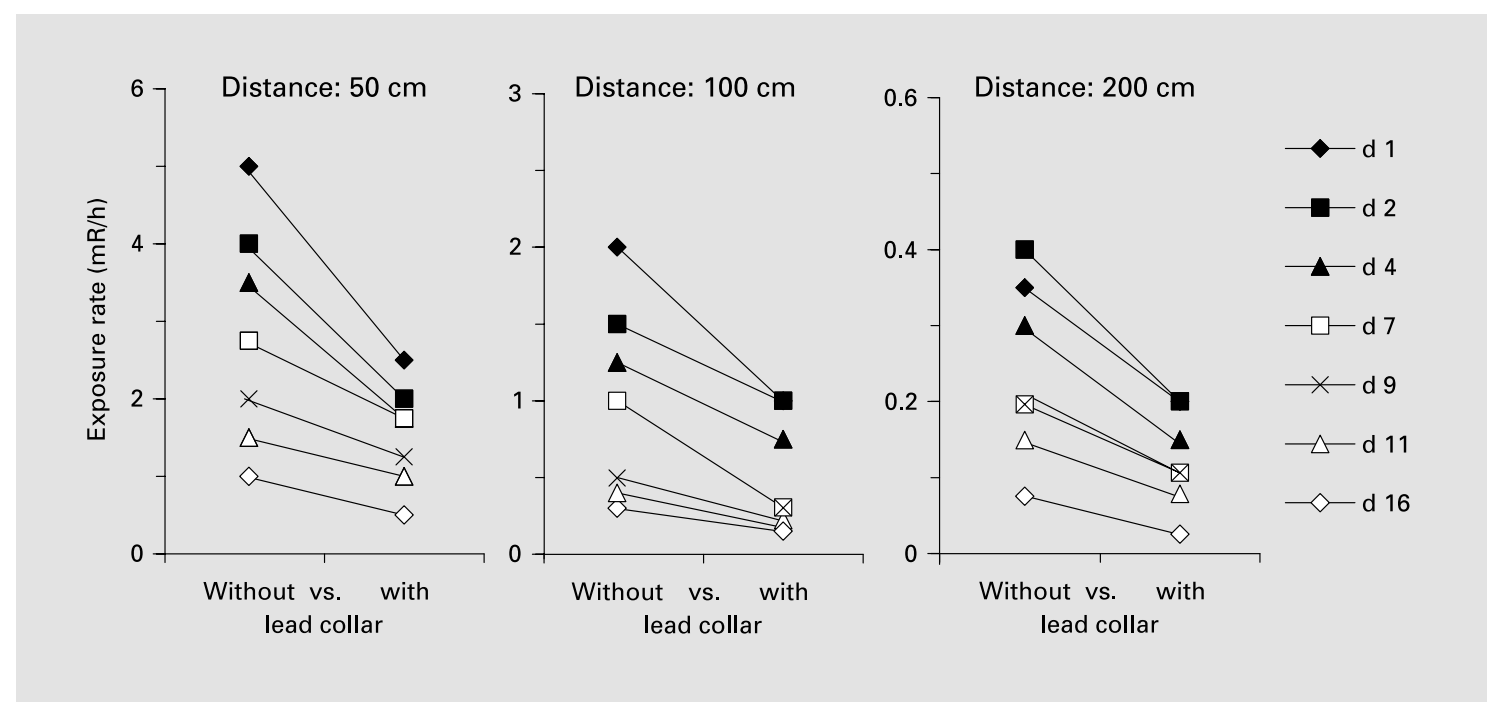

Fig. 2. Radiation exposure rates $(\mathrm{mR} / \mathrm{h})$ measured at 50,100 and $200 \mathrm{~cm}$ from a patient on various days (1-16) after ${ }^{131}$ I treatment without or with the lead collar in place (background rate $<1 \mu \mathrm{R} / \mathrm{h}$ ).

and $200 \mathrm{~cm}$ distance from the patient's neck anteriorly and horizontally using a calibrated radiation survey meter (Atomic Products Corp. Survey Meter Model 069-701, USA). Paired readings with the patient wearing the lead collar and not wearing it were obtained. The measurements were adjusted for the amount of ${ }^{131}$ I activity localized in the thyroid obtained by multiplying the radioactivity administered with the radioiodine uptake. Radiation exposure was then expressed as microröntgens per hour per megabecquerel (or microröntgens per hour per millicurie) localized in the thyroid with and without the lead collar in place. Comparison of the measurements with and without the lead collar was done using the paired $t$ test. The level of significance was $\mathrm{p}<0.05$.

\section{Results}

The collar did not cause any skin reaction or any problem with draining blood from the neck veins. Only 1 patient complained of the weight of the collar but was nevertheless willing to wear the device as instructed. The overall acceptance of the individual patients to wear the new device was excellent.

The average ratio of the exposure from the patients with to that without the collar was $0.54 \pm 0.12$, with $n=$ 200 pairs of measurement (actual measurements in all patients not shown). The measured reduction in the exposure rate by wearing the lead collar was highly significant in individual patients and in the group as a whole (paired $t$ test, $\mathrm{p}<0.001)$. In figure 2 , an example of the radiation exposure measurements is given in a patient showing the reduction of the exposure rate with and without the lead collar at various distances $(50,100,200 \mathrm{~cm})$ and times (1-16 days) after treatment.

A summary of the approximate radiation exposure per unit radioactivity (megabecquerel or millicurie) localized in the thyroid at various distances and times after ${ }^{131} \mathrm{I}$ administration is shown in table 2 .

\section{Discussion}

Limiting radiation exposure from patients treated with ${ }^{131}$ I is a controversial issue, especially in cases receiving radioactive doses not requiring confinememt in hospital by law such as patients with hyperthyroidism. There is however a general consensus among workers in the field to reduce exposure from those patients by taking measures restricting their contact with other individuals $[2,8$, 17]. A variety of posttreatment protocols have been reported reflecting the attitude towards exposure from a relatively permissive to the most stringent protocol $[3,5$, $9,16]$. Examples of the restrictions imposed are separate sleeping arrangement for a variable period of time and limited contact with high-risk groups such as pregnant women and children [13, 14].

Our data indicate relatively high levels of exposure to the radiation emanating from the patient at distances less that $2 \mathrm{~m}$ during the first week after the treatment (table 2). 
Table 2. Radiation exposure rate per unit radioactivity: average, minimum and maximum, localized in the thyroid without and with the lead collar in place from day 1 to 21 after treatment with ${ }^{131}$ I

\begin{tabular}{|c|c|c|c|c|c|c|c|}
\hline \multirow[t]{2}{*}{ Day } & \multirow[t]{2}{*}{$\mathrm{n}$} & \multicolumn{2}{|c|}{ Rate at $50 \mathrm{~cm}, \mu \mathrm{R} / \mathrm{h} / \mathrm{MBq}$} & \multicolumn{2}{|c|}{ Rate at $100 \mathrm{~cm}, \mu \mathrm{R} / \mathrm{h} / \mathrm{MBq}$} & \multicolumn{2}{|c|}{ Rate at $200 \mathrm{~cm}, \mu \mathrm{R} / \mathrm{h} / \mathrm{MBq}$} \\
\hline & & without & with & without & with & without & with \\
\hline 1 & 9 & $29,12,63$ & $14,6,29$ & $9,4,19$ & $5,2,10$ & $2,0.8,4$ & $1,0.5,2$ \\
\hline 2 & 3 & $30,10,65$ & $13,5,26$ & $4,2,5$ & $2,1.3,3$ & $1,0.6,1$ & $0.5,0.4,0.7$ \\
\hline 3 & 3 & $15,11,17$ & $7,5,9$ & $5,2,6$ & $2,1,3$ & $1,0.9,2$ & $0.7,0.5,1$ \\
\hline 4 & 10 & $15,3,27$ & $8,2,12$ & $5,0.9,9$ & $3,0.9,6$ & $2,0.2,5$ & $0.8,0.1,2$ \\
\hline 5 & 4 & $8,5,13$ & $4,3,6$ & $3,1,5$ & $1,0.8,3$ & $0.5,0.2,1.3$ & $0.3, \mathrm{BG}, 0.6$ \\
\hline 6 & 6 & $9,4,17$ & $5,2,9$ & $3,0.8,6$ & $1.5,0.3,2.6$ & $0.7,0.2,1.2$ & $0.4,0.1,0.8$ \\
\hline 7 & 8 & $8,3,14$ & $5,2,8$ & $3,1,4$ & $1.5,0.4,2.6$ & $0.7,0.2,1.2$ & $0.4,0.1,0.9$ \\
\hline 8 & 5 & $9,5,13$ & $5,3,6$ & $3.5,1,6$ & $1.6,0.5,2.4$ & $0.9,0.3,1.7$ & $0.5,0.2,0.7$ \\
\hline 9 & 1 & 4.8 & 3 & 1.2 & 0.6 & 0.5 & 0.24 \\
\hline 10 & 1 & 5 & 3.4 & 1.7 & 0.9 & 0.5 & 0.2 \\
\hline 11 & 4 & $5,1,10$ & $3,0.6,5$ & $1.3,0.4,2$ & $0.6,0.3,1$ & $0.5,0.1,0.7$ & $0.3,0.1,0.5$ \\
\hline 12 & 4 & 4 & $2,1,3$ & $1,0.8,1.3$ & $0.5,0.5,0.6$ & $0.2,0.1,0.3$ & $0.1,0.1,0.2$ \\
\hline 13 & 2 & $6,4,7$ & $4,2.5,5$ & $1.7,1.7,1.7$ & $0.9,0.8,1$ & $0.5,0.4,0.5$ & 0.3 \\
\hline 14 & 3 & $6,3,9$ & $3,1.5,4$ & $1.7,1.3,2$ & $0.8,0.5,1.3$ & $0.6,0.4,0.9$ & $0.2,0.2,0.2$ \\
\hline 15 & ND & & & & & & \\
\hline 16 & 1 & 2.4 & 1 & 0.7 & 0.4 & 0.2 & 0.1 \\
\hline 17 & ND & & & & & & \\
\hline 18 & 1 & 1 & 0.5 & 0.4 & 0.2 & 0.2 & 0.1 \\
\hline 19 & 1 & 1 & 0.4 & 0.4 & 0.2 & 0.1 & 0.1 \\
\hline 20 & ND & & & & & & \\
\hline 21 & 3 & $1.4,1,1.7$ & $0.8,0.6,0.9$ & $0.3, \mathrm{BG}, 0.5$ & $0.1, \mathrm{BG}, 0.2$ & $0.03, \mathrm{BG}, 0.1$ & $0.03, \mathrm{BG}, 0.1$ \\
\hline Sum & & 160 & 82 & 45 & 23 & 12 & 6 \\
\hline
\end{tabular}

$\mathrm{n}=$ Number of patients measured; $\mu \mathrm{R} / \mathrm{h} / \mathrm{MBq}$ : multiply by 37 for conversion to $\mu \mathrm{R} / \mathrm{h} / \mathrm{mCi} ; \mathrm{BG}=$ background level $<1 \mu \mathrm{R} / \mathrm{h} ; \mathrm{ND}=$ not done.

However, the exposure to radiation during that period would be limited if radiation protection protocols were followed depending on the policy of the institution delivering the treatment. The lead collar would additionally reduce radiation exposure especially at short distances from the patient and over the time period of its use by a factor of 2. Accordingly and by simple extrapolation, a reduction of the radiation dose to the general public of up to about $50 \%$ would be achieved by using the lead collar. The extent of reduction would depend on the circumstances of the individual patient and the radiation protection policy of the treating institution.

The benefit of wearing the lead collar is particularly significant when the patient cannot abide by the restrictive measures as given in table 1 , especially those related to contact with other individuals. This is especially notable in the case of a mother with young children where close-range exposure cannot be avoided. In such a situation, the patient can wear the collar continuously except while sleeping preferably in a separate room with no other members of the family present. The duration of collar use would be for the first week but could be extended to the end of the second week if very-short-range exposure $(0.5-1 \mathrm{~m})$ was occurring extensively such as in the case of a mother with very young children (less than 2 years). The same guidelines would be applicable to patients in a similar situation to the above such as workers in nurseries or child care centers. In cases where a prolonged presence of the patient in proximity to other mainly adult work colleagues is found, the use of the collar would be advised if there was a high likelihood of contact with pregnant women. The duration of use would be for the first week after treatment since the exposure drops significantly in the second week, especially for distances of more than $1 \mathrm{~m}$. In the examples above, the new device is acting as the only protective means to reduce exposure to people in contact with the patient.

The use of the lead collar may be beneficial in those situations where it would be favorable to avoid random exposure of the general public to radiation such as casual 
contact on public transportation or in shopping facilities. The significance of this reduction, however, remains uncertain and requires further study.

Wearing of the lead collar provides a psychological reassurance to family members and the public that the lead collar is helpful in reducing radiation emitted by the patient. However, the desired reduction using the lead collar could be offset by the patient abandoning all other restrictive measures after receiving the radioactive treatment. An approach integrating certain restrictions on the radioactive patient along with judicial use of the lead collar would provide the optimal plan for reducing unnecessary irradiation to the patient's surroundings.

\section{Conclusion}

A significant decrease in the exposure has been shown which would be beneficial especially in patients in whom the restrictive methods after treatment do not strictly apply.

\section{Acknowledgements}

Part of this research work was presented as a poster with the same title at the Congress of the European Association of Nuclear Medicine in Barcelona, Spain, in 1999.

\section{References}

1 Gittoes NJ, Franklyn JA: Hyperthyroidism: Current treatment guidelines. Drugs 1998;55: 543-553.

2 Pochin EE, Kermode JC: Protection problems in radionuclide therapy: The patient as a gamma-radiation source. Br J Radiol 1975;48:299_ 305.

3 Culver CM, Dworkin HJ: Radiation safety considerations for post-iodine-131 hyperthyroid therapy. J Nucl Med 1991;32:169-173.

4 Barrington SF, O'Doherty MJ, Kettle AG, Thomson WH, Mountford PJ, Burrell DN, Farrell RJ, Batchelor S, Seed P, Harding LK: Radiation exposure of the families of outpatients treated with radioiodine (iodine-131) for hyperthyroidism. Eur J Nucl Med 1999;26: 686-692.

5 Reiners C, Lassmann M: Radioiodine ( $\left.{ }^{131} \mathrm{I}\right)$ treatment of hyperthyroidism: Radiation protection and quality assurance. Eur J Nucl Med 1999;26:683-685.

6 Doi SA, Loutfi I, Al-Shoumer KA: A mathematical model of optimized radioiodine-131 therapy of Graves' hyperthyroidism. BMC Nucl Med 2001;1:1.

7 Recommended dose limits; 1990 Recommendations of the International Commission on Radiological Protection. ICRP Publication 60, Annal of the ICRP. New York, Pergamon Press, 1991, vol 21, No 1-3, pp 46, 72.
8 Mountford PJ, O’Doherty MJ: Restrictions following iodine-131 treatment - A time for change or more data required? Eur J Nucl Med 1994;21:903-905.

9 US Nuclear Regulatory Commission: Medical use of byproduct material (10 CFR Part 35). Federal Register (67 FR 20249) April 24, 2002.

10 Siegel JA: Revised Nuclear Regulatory Commission regulations for release of patients administered radioactive materials: Outpatient iodine-131 anti-B1 therapy. J Nucl Med 1998; 39(suppl):28S-33S.

11 Working Party of the Radiation Protection Committee of the British Institute of Radiology: Patients leaving hospital after administration of radioactive substances. $\mathrm{Br} \mathrm{J}$ Radiol 1999;72:121-125.

12 Wasserman HJ, Klopper JF: Analysis of radiation doses received by the public from ${ }^{131} \mathrm{I}$ treatment of thyrotoxic outpatients. Nucl Med Commun 1993;14:756-760.

13 Mountford PJ, Steele HR: Fetal dose estimates and the ICRP abdominal dose limit for occupational exposure of pregnant staff to technetium- $99 \mathrm{~m}$ and iodine-131 patients. Eur J Nucl Med 1995;22:1173-1179.

14 Mountford PJ, O’Doherty MJ: Exposure of critical groups to nuclear medicine patients. Appl Radiat Isot 1999;50:89-111.
15 Weber PJ, Castronovo FP: A software application to estimate exposure around ${ }^{131}$ I cancer and hyperthyroid patients. Nucl Med Commun 1996;17:8-14.

16 Monsieurs M, Thierens H, Dierckx RA, Casier K, De Baere E, De Ridder L, De Saedeleer C, De Winter $H$, Lippens $M$, van Imschoot $S$, Wulfrank D, Simons M: Real-life radiation burden to relatives of patients treated with iodine-131: A study in eight centres in Flanders (Belgium). Eur J Nucl Med 1998;25:13681376.

17 Mathieu I, Caussin J, Smeesters P, Wambersie A, Beckers C: Recommended restrictions after ${ }^{131}$ I therapy: Measured doses in family members. Health Phys 1999;76:129-136.

18 Nichizawa K, Ohara K, Ohshima M, Maekoshi $\mathrm{H}$, Orito T, Watanabe T: Monitoring of iodine excretions and used material of patients treated with ${ }^{131}$ I. Health Phys 1980;38:467-481.

19 Demir M, Kabasakal L, Onsel C: Evaluation of external radiation exposure rate from radioiodine-treated hyperthyroid patients and radiation safety considerations. Nucl Med Commun 1996;17:692-695.

20 O'Hare NJ, Murphy D, Malone JF: Thyroid dosimetry of adult European populations. Br J Radiol 1998;71:535-543.

21 Berg GE, Michanek AM, Holmberg EC, Fink M: Iodine-131 treatment of hyperthyroidism: Significance of effective half-life measurements. J Nucl Med 1996;37:228-232. 